\title{
Surface Graft Polymerization of Binary Monomers Maleic Anhydride/n-Butyl Vinyl Ether on the Polypropylene Film via Two-Step Method
}

\author{
Chang-Min XING, ${ }^{*, * *}$ Jian-Ping DENG, ${ }^{*, * *}$ and Wan-Tai YANG ${ }^{*, * *, \dagger}$ \\ *Department of Polymer Science, Beijing University of Chemical Technology, \\ Beijing 100029, China \\ ${ }^{* *}$ Key Laboratory of Science and Technology of Controllable Chemical Reactions, \\ Ministry of Education, Beijing 100029, China
}

(Received January 20, 2003; Accepted June 9, 2003)

\begin{abstract}
Maleic anhydride (MAH) was readily grafted onto polypropylene (PP) film by means of introducing the electron donor monomer $n$-butyl vinyl ether ( $n$-BVE), via two-step method. In the first step, the semibenzopinacol (SBP) groups were introduced onto the film surface under UV irradiation, and they were subsequently detected by both gravimetric method and UV spectra. In the second step, the "dormant" SBP groups were activated and cleaved from the substrate under heat, resulting in the formation of surface free radicals (grafting points) and the immediate initiation of graft polymerization; the temperature required for this process was found to be around $80^{\circ} \mathrm{C}$. The grafting results show that the grafting yield $\left(Y_{\mathrm{g}}\right)$ of two-step method can be much higher than that of one-step method, and that the novel electron donor-acceptor grafting system is much more effective than that of pure MAH. Properly speaking, the $Y_{\mathrm{g}}$ value could reach as high as $180 \%$ within $1 \mathrm{~h}$, and the density of polar groups $\left(D_{\mathrm{p}}\right)$ on the substrate was $49.5 \mu \mathrm{mol} \mathrm{cm}^{-2}$ correspondingly. Besides, the maximum $Y_{\mathrm{g}}$ can be always attained at 1:1 molar feed ratio of MAH to BVE, indicating that the facile grafting process is attributed to the formation of charge transfer complex (CTC) between the binary monomers. Another significant result is that the good affinity of solvent towards substrate, and especially the desirable solubility for the graft chains are both preferable to heterogeneous graft polymerization. In addition, some phenomena characterizing "living" graft polymerization were revealed in this particular grafting system, which is expected to favor the control of the length and chemical structure of the graft chains.

KEY WORDS Surface Modification / Graft Polymerization / Maleic Anhydride / $n$-Butyl Vinyl Ether / Charge Transfer Complex /
\end{abstract}

Polyolefin, including polyethylene (PE), polypropylene (PP), ethylene-propylene elastomer (EPR), etc., have been the most widely used commercial polymers, due to their excellent combination of chemical and physical properties along with low cost, superior processability. ${ }^{1,2}$ But, all the polyolefinic materials lack polar groups on their macromolecular backbones, thus leading to low surface energy, poor adhesion, undesirable compatibility and many other problems, which have prevented their even wider usage. So many researchers have focused on the introduction of polar groups into the existing polyolefin for years, ${ }^{3-5}$ mostly by graft polymerization, which is initiated by various means, such as chemical reagents, ${ }^{6}$ electronic beam, ${ }^{7,8}$ $\gamma$-ray, plasma, ${ }^{9} \mathrm{UV}$ light, shear force, ${ }^{10}$ etc.

The photoinduced graft polymerization has been a popular method for surface modification or functionalization of polyolefinic materials due to its remarkable advantages, ${ }^{11-13}$ such as weak penetrability without damaging the bulk property, mild reaction conditions and low cost of operation. With regard to the monomer for photografting polymerization, the vinyl monomers are widely used, ${ }^{14,15}$ including acrylic acid (AA), methacrylic acid (MAA), methyl methacrylate (MMA), styrene (St), acrylamide (AM), acrylonitrile (AN), vinyl acetate (VAc), etc. Maleic anhydride (MAH), however, was seldom used in surface photografting, although it is commonly used for melt grafting. ${ }^{16,17}$ In fact, MAH used as a monomer for surface modification has significant advantages: 1) The anhydride group in MAH molecule has fairly strong polarity and each can be hydrolyzed into two carboxyl groups, which will effectively increase the density of the polar groups on the material surface; 2) The anhydride group can undergo a number of reactions with other organic groups due to its high activity, which will make the grafted polymer possess many potential functions. But, MAH is difficult to homopolymerize under usual conditions due to its great steric hindrance, as commonly known, which in turn leads to very low grafting yield. Previously, H. Kubota, et al. ${ }^{18}$ pointed out that the monomers difficult to/cannot be

†Part 3 of a series "Surface graft polymerization based on charge transfer complex of maleic anhydride", for parts 1 and 2 see ref 19 and 26.

${ }^{\dagger \dagger}$ To whom correspondence should be addressed (E-mail: yangwt@mail.buct.edu.cn). 
grafted will readily undergo graft reactions by means of introducing the suitable comonomer. Given that the electron donor monomer $n$-butyl vinyl ether (n-BVE) can form charge transfer complex (CTC) with MAH and they will consequently undergo alternating copolymerization, the grafting system of binary monomers MAH/BVE was designed.

In our previous work, ${ }^{19} \mathrm{MAH} / \mathrm{BVE}$ had been facilely photografted onto PP film via one-step method, and the property (e.g., hydrophilicity) of the film surface was effectively improved. In that method, the reactive solution composed of monomer and photoinitiator was coated on the film surface first, then put under UV radiation to perform graft polymerization. Due to the limited amount of monomer added, the grafting yield cannot be high (averagely less than 1\%), although it is enough for surface modification of the polymeric materials. However, for functional membranes or fibers used for ion exchange, adsorption, immobilization of enzyme/cells, etc., it is required that the density of polar groups on the material surface must be rather high. Formerly, we put forward a novel twostep graft polymerization, ${ }^{20}$ which can facilely achieve the above requirement. Firstly, some particular groups are introduced onto the film surface under UV irradiation; secondly, these introduced "dormant" groups are activated and cleaved from the substrate under heat or successive UV irradiation, thus the surface free radicals (grafting points) are formed and the graft polymerization is consequently initiated in monomer solution. H. M. Ma, C. N. Bowman, et al. ${ }^{21}$ once conducted the second-step grafting under successive UV irradiation. Here a novel thermoactivating graft polymerization on the pre-irradiated film was investigated intensively and some significant results were obtained.

\section{EXPERIMENTAL}

\section{Materials}

Commercial casting polypropylene (CPP) film, $30 \mu \mathrm{m}$ thick, with transmittance close to $100 \%$ for UV radiation (200-400 $\mathrm{nm}$ ), was used as the substrate, which was first cut into circular shape of about $40 \mathrm{~mm}$ in diameter and then subjected to Soxhlet extraction with acetone for $24 \mathrm{~h}$ to remove the additives and impurities.

Maleic anhydride (MAH) and acrylic acid (AA) were purified by distillation before use, and $n$-butyl vinyl ether ( $n$-BVE) was analytical grade, used without further purification. The photoinitiator benzophenone (BP) was analytical grade, used without further purification. The solvents acetic anhydride (AAH) and acetone $(\mathrm{AC})$, were analytical grade, used without further purification.

\section{UV Pre-irradiation Procedure}

In the UV pre-irradiation step, both the apparatus and the operation were just like those in the photografting process, ${ }^{13,19}$ except that only BP is present in the reactive solution instead of the combination of $\mathrm{BP}$ and monomer. After irradiation, the film was clearly washed with acetone to remove the residual photoinitiator and then dried to constant weight at room temperature. The weight gain of the pre-irradiated film was determined by an analytical balance (Sartorius BP211D, Germany) with the accuracy of $0.01 \mathrm{mg}$.

\section{Graft Polymerization Procedure}

The pre-irradiated films were put into a solution containing the binary monomers MAH and BVE $(50 \mathrm{~mL})$, which had been purged with $\mathrm{N}_{2}$ for 30 min to remove $\mathrm{O}_{2}$ dissolving in the system. The reaction temperature was controlled by a water bath and the graft polymerization was conducted under $\mathrm{N}_{2}$ atmosphere all the time. After reaction, the grafted films were taken out and Soxhlet-extracted with acetone for $8 \mathrm{~h}$ to remove the homopolymer adhered on the substrate (in fact, the ungrafted polymer in this system is copolymer, but we still conventionally call it homopolymer, et seq.), and finally dried to constant weight in a vacuum oven at $60^{\circ} \mathrm{C}$. The weight gain of the films was also determined by the analytical balance.

The percent graft of BP on the substrate $\left(G_{\mathrm{BP}}\right)$, surface density of the introduced "dormant" groups $\left(D_{\mathrm{BP}}\right)$, grafting yield $\left(Y_{\mathrm{g}}\right)$, surface density of the polar groups $\left(D_{\mathrm{p}}\right.$, after the anhydride groups hydrolyzed into carboxyl groups), grafting efficiency $\left(E_{\mathrm{g}}\right)$ and average graft chain length $\left(L_{\mathrm{c}}\right)$ were obtained by the following definitions:

$$
\begin{aligned}
& G_{\mathrm{BP}}=W_{\mathrm{BP}} / W_{\mathrm{f}} \times 100 \% \\
& D_{\mathrm{BP}}=W_{\mathrm{BP}} /\left(M W_{\mathrm{BP}} \times S\right) \\
& Y_{\mathrm{g}}=W_{\mathrm{g}} / W_{\mathrm{f}} \times 100 \% \\
& D_{\mathrm{p}}=W_{\mathrm{g}} /(M W \times S) \times 2 \\
& E_{\mathrm{g}}=W_{\mathrm{g}} /\left(W_{\mathrm{p}}+W_{\mathrm{g}}\right) \times 100 \% \\
& L_{\mathrm{c}}=\left(W_{\mathrm{g}} / M W\right) /\left(W_{\mathrm{BP}} / M W_{\mathrm{BP}}\right)
\end{aligned}
$$

where $W_{\mathrm{BP}}$ is the weight gain of the film after preirradiation; $W_{\mathrm{f}}$ is the weight of the blank film; $M W_{\mathrm{BP}}$ is the molecular weight of BP; $S$ is the surface area of the film; $W_{\mathrm{g}}$ is the weight of the graft chains formed; $M W$ is the molecular weight of the graft chain unit (MAHalt-BVE); $W_{\mathrm{p}}$ is the homopolymer yielded in the solution. 


\section{UV Spectra}

The UV spectra of the pre-irradiated films, as well as those of the grafted films, were measured by UV-vis Spectrometer (GBC Cintra 20, Australia).

\section{FT-IR Spectra}

The infrared (IR) spectra of the grafted films were measured by Fourier transform infrared spectrometer (ThermoNicolet Nexus 670, USA). The IR spectra of the blank PP film, maleic anhydride, poly(butyl vinyl ether) and poly(acrylic acid) were also presented as reference.

\section{RESULTS AND DISCUSSION}

\section{Introduction of "Dormant" Groups onto Film Surface}

BP is a typical hydrogen abstractable photoinitiator (Norrish II type) popularly used in the photografting and photocross-linking processes. When irradiated by $\mathrm{UV}, \mathrm{BP}$ is excited to the single state $\left(\mathrm{BP}^{\mathrm{S}}\right)$ and immediately relaxes to the stable triplet state $\left(\mathrm{BP}^{\mathrm{T}}\right)$ through intersystem crossing (ISC), then $\mathrm{BP}^{\mathrm{T}}$ abstracts hydrogen from the surface of the polymeric substrate-the hydrogen donor. ${ }^{22,23}$ Thus the surface free radicals (I) and semibenzopinacol (SBP) radicals (II) are generated, as expressed in Scheme 1.

Due to the steric hindrance and $\pi$-conjugate effect, the SBP radicals would rather combine with the surface radicals than undergo coupling reactions each other, ${ }^{21}$ thus the pre-irradiated film (III) is formed. Such preirradiated film can be put aside for several days or months without losing the activity, so the SBP groups introduced onto the film surface can be called "dormant" groups. The existence of these groups was determined by both gravimetric method and UV spectra, and the results are shown in Table I and Figure 1.

It can be seen from Table I that the weight gain of the PP film distinctly occurred under UV irradiation in the presence of BP. This indicated that the SBP groups were successfully introduced and chemically bonded onto the film surface. The data in Table I also show that the percent graft of BP $\left(G_{\mathrm{BP}}\right)$ and the corresponding density of SBP groups $\left(D_{\mathrm{BP}}\right)$ varied under various reaction conditions. This means that the density of grafting points on the film surface can be controlled by adjusting the pre-irradiation conditions, such as photoinitiator concentration and UV irradiation time in the first step. It is worthy of pointing out that at high values of $G_{\mathrm{BP}}$, some SBP groups may immobilize inside the film, besides on the surface and subsurface. This is easy to be understood from the fact that some photoinitiator BP would diffuse into the substrate in the medium of acetone, a solvent well affinitive towards polyolefin ma-

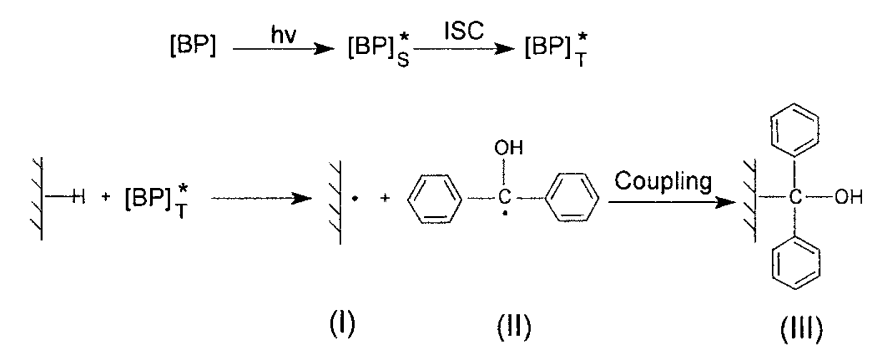

Scheme 1. Introduction of "dormant" groups onto film surface based on BP photoreduction.

Table I. Amount of the introduced SBP groups on the film surface under various pre-irradiation conditions $\mathrm{s}^{\mathrm{a}}$

\begin{tabular}{cccc}
\hline $\begin{array}{c}\text { BP conc. } \\
(\mathrm{wt} \%)\end{array}$ & $\begin{array}{c}\text { Irradiation } \\
\text { time }(\mathrm{s})\end{array}$ & $\begin{array}{c}G_{\mathrm{BP}} \\
(\%)\end{array}$ & $\begin{array}{c}D_{\mathrm{BP}} \\
\left.(\mu \mathrm{mol} \mathrm{cm})^{-2}\right)\end{array}$ \\
\hline 5 & 60 & 0.11 & 0.017 \\
5 & 120 & 0.27 & 0.041 \\
10 & 60 & 0.18 & 0.028 \\
10 & 120 & 0.34 & 0.052 \\
\hline
\end{tabular}

${ }^{\mathrm{a}}$ Solvent, acetone; reaction temperature, $25^{\circ} \mathrm{C}$;

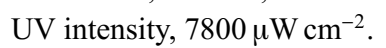

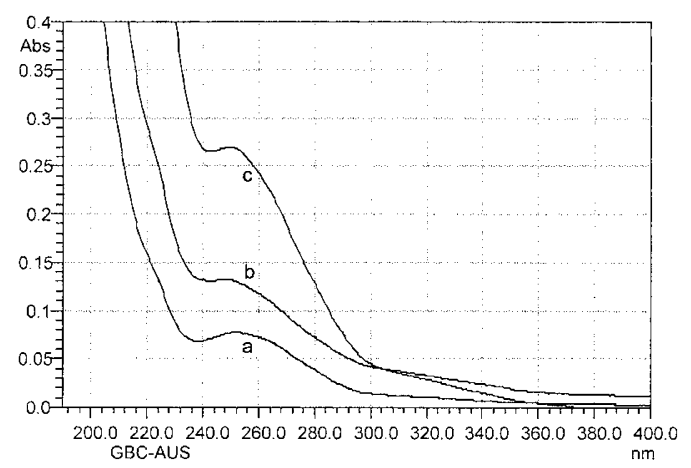

Figure 1. UV spectra of the pre-irradiated films prepared under various reaction conditions. a, $5 \mathrm{wt} \%$ of BP, $60 \mathrm{~s} ; \mathrm{b}, 10 \mathrm{wt} \%$ of BP, $60 \mathrm{~s} ; \mathrm{c}, 10 \mathrm{wt} \%$ of BP, $120 \mathrm{~s}$.

terials. As for the PP substrate used in this study, it has been recognized that the graft reaction takes place mostly at the tertiary carbon positions ${ }^{24}$ because the tertiary hydrogens on the backbone are preferentially abstracted by BP due to the weaker $\mathrm{C}-\mathrm{H}$ bond at this site.

The UV spectra in Figure 1 show that a specific absorption appears at around $254 \mathrm{~nm}$, which is a remarkable characteristic of the SBP groups. This is another evidence confirming the successful introduction of SBP radicals onto film surface in the first step.

\section{Thermoactivating Surface Graft Polymerization}

When heated or under successive UV irradiation, the "dormant" SBP groups on the pre-irradiated film would be easily activated and split off the substrate due to the weak bond energy; thus the surface free radicals are again formed and subsequently initiate graft polymerization. ${ }^{20,21}$ This process was illustrated in Scheme 2. 


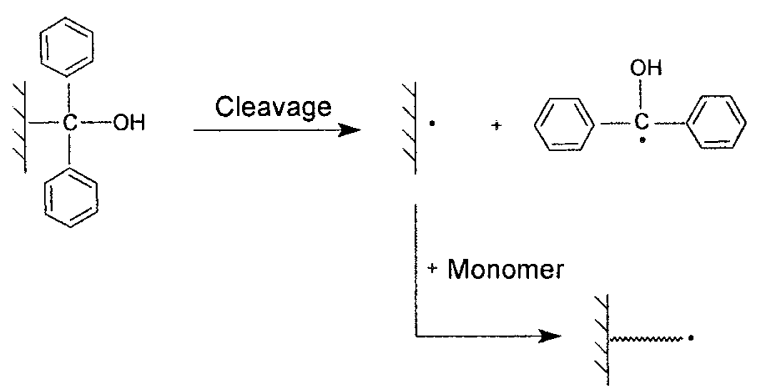

Scheme 2. Mechanism of the thermoactivating graft polymerization on the pre-irradiated film.

Table II. Performance of the surface graft polymerization of $\mathrm{MAH} / \mathrm{BVE}$ on the pre-irradiated films ${ }^{\mathrm{a}}$

\begin{tabular}{ccccc}
\hline $\begin{array}{c}D_{\mathrm{BP}} \\
\left(\mu \mathrm{mol} \mathrm{cm} \mathrm{cm}^{-2}\right)\end{array}$ & $\begin{array}{c}{[\mathrm{MAH}] /} \\
{[\mathrm{BVE}]^{\mathrm{b}}}\end{array}$ & $\begin{array}{c}\text { Reaction } \\
\text { time }(\min )\end{array}$ & $\begin{array}{c}Y_{\mathrm{g}} \\
(\%)\end{array}$ & $\begin{array}{c}D_{\mathrm{p}} \\
\left.(\mu \mathrm{mol} \mathrm{cm})^{-2}\right)\end{array}$ \\
\hline 0.052 & $2 / 2$ & 60 & 30.5 & 8.4 \\
0.052 & $2 / 2$ & 120 & 81.7 & 22.5 \\
0.081 & $2 / 2$ & 60 & 60.4 & 16.6 \\
0.081 & $2 / 2$ & 120 & 133.4 & 36.7 \\
0.081 & $0 / 4^{\mathrm{c}}$ & 180 & 0 & 0 \\
0.081 & $4 / 0^{\mathrm{d}}$ & 180 & 0.8 & 0.2 \\
\hline
\end{tabular}

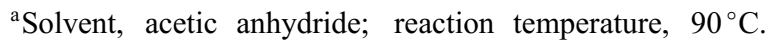

${ }^{b}$ Molar feed ratio of MAH to BVE, the total concentration is

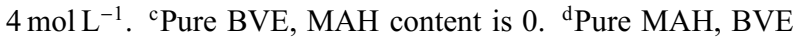
content is 0 .

The general performances of the surface graft polymerization of $\mathrm{MAH} / \mathrm{BVE}$ on the pre-irradiated films were summarized in Table II.

The data in Table II show that when MAH content was 0 , pure BVE could not undergo graft polymerization; while pure MAH could perform graft reaction to some degree; but, if the molar feed ratio of MAH to BVE was $1: 1$, the graft polymerization could readily proceed. These results indicate that the graft polymerization of MAH can be remarkably promoted by means of applying the electron donor-acceptor binary monomer system. The detailed results and mechanism of the effect of MAH/BVE ratio are presented in the following section of this article.

Also from Table II, it can be seen that the grafting yield $\left(Y_{\mathrm{g}}\right)$ of this two-step method is much higher than that of one-step method (averagely less than 1\%), and correspondingly, the density of the polar groups $\left(D_{\mathrm{p}}\right)$ on the substrate can be fairly high. Moreover, both $Y_{\mathrm{g}}$ and $D_{\mathrm{p}}$ values vary along with the $D_{\mathrm{BP}}$ values and the reaction time, which means that the grafting results can be controlled by adjusting the pre-irradiation conditions in the first step and the grafting conditions in the second step. These results above indicate that the novel graft polymerization of MAH/BVE via two-step method is a desirable approach for surface functionalization of the polyolefinic materials.

For the same reason, the SBP radicals hardly have a

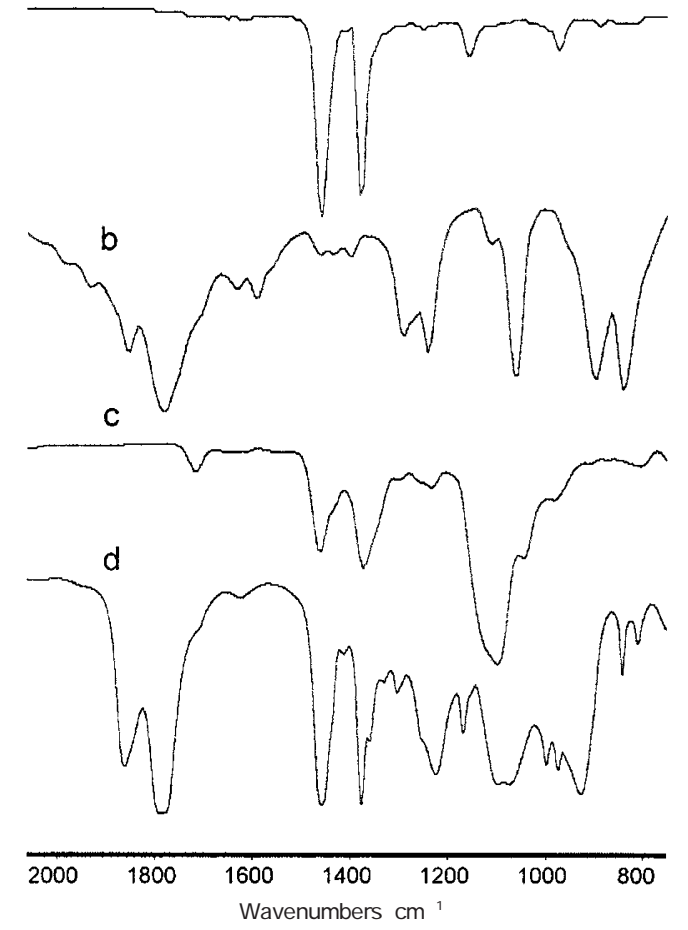

Figure 2. FT-IR Spectra of a, blank PP film; b, maleic anhydride, c, poly(butyl vinyl ether) and d, grafted PP film.

initiating ability, thus the homopolymerization in the solution can be effectively inhibited and high grafting efficiency is expected to achieve. ${ }^{21}$ In this particular grafting system, however, because the formed CTC between the binary monomers can be thermally initiated, ${ }^{25}$ the homopolymerization occurs to some extent, especially at high monomer concentration. The experimental results show that $E_{\mathrm{g}}$ is $50-90 \%$ at the concentration lower than $2.0 \mathrm{~mol} \mathrm{~L}^{-1}$, while $E_{\mathrm{g}}$ declines gradually to less than $20 \%$ at higher concentrations (e.g., $6 \mathrm{~mol} \mathrm{~L}^{-1}$ ).

The successful grafting of both MAH and BVE was further confirmed by FT-IR spectra, as shown in Figure 2.

It can be seen that some specific vibration absorptions appeared in the grafted PP film compared with that of the blank PP film. The absorptions at $1785 \mathrm{~cm}^{-1}$ and $1860 \mathrm{~cm}^{-1}$ indicate the existence of anhydride group in $\mathrm{MAH}$ structure, and the absorption at $1102 \mathrm{~cm}^{-1}$ indicates the existence of ether bond in BVE structure.

\section{Effect of the Feed Ratio of MAH to n-BVE}

In this study, the electron donor monomer $n$-BVE was introduced into the reaction system to promote the graft polymerization of MAH. So the effect of the feed ratio of MAH to BVE was investigated intensively and the results are shown in Figure 3.

There are three obvious characteristics exhibited in 


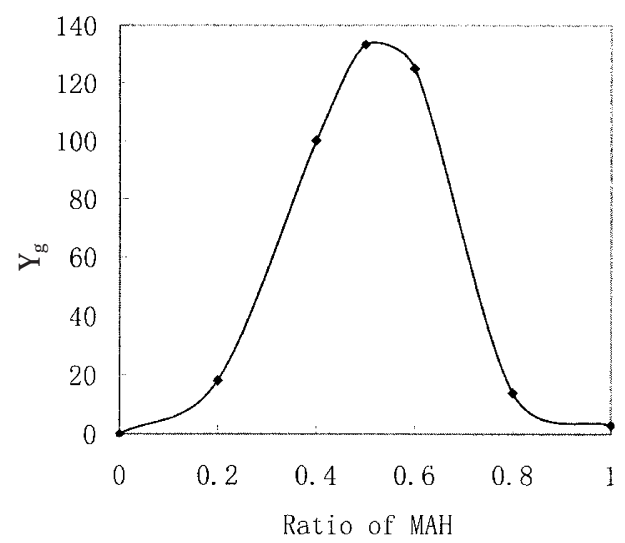

Figure 3. Effect of the molar feed ratio of MAH to BVE on

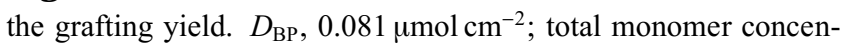
tration, $4 \mathrm{~mol} \mathrm{~L}^{-1}$; solvent, acetic anhydride; reaction temperature, $90^{\circ} \mathrm{C}$; reaction time, $120 \mathrm{~min}$.

Figure 3. (1) The grafting yield reached the maximum value when the molar feed ratio of MAH to BVE was 1:1. (2) When MAH content was 0 , the value of $Y_{\mathrm{g}}$ was 0 , which indicated that BVE itself could not undergo graft polymerization. (3) When BVE content was 0 , MAH itself could perform graft polymerization to some degree. These results were just similar to those in the one-step method. ${ }^{19}$

Since BVE is a typical monomer for cationic polymerization, as commonly known, it is impossible to undergo radical homopolymerization. Due to the great steric hindrance, MAH is difficult to perform homopolymerization under usual conditions and only some short graft chains could be obtained. But MAH, as an electron acceptor $(e=+2.25)$, and the introduced $\mathrm{BVE}$, as an electron donor $(e=-1.6)$, prefer to form charge transfer complex (CTC), ${ }^{25}$ and consequently they readily undergo alternating copolymerization. The CTC between the electron donor-acceptor molecules can be detected by various methods such as UV spectra and nuclear magnetic resonance (NMR), as discussed in detail in our previous article. ${ }^{26}$ So far, the formation of CTC has been popularly considered as a major mechanism of alternating copolymerization, although there still exists some controversy on this issue. In this study, we have just implanted the alternating copolymerization into the surface graft polymerization field by means of introducing the electron donor monomer $\mathrm{BVE}$, achieving the grafting of MAH onto the polymer substrate facilely.

\section{Effect of the Reaction Temperature}

The primary requirement for the graft polymerization in the second step is that the SBP groups split off the pre-irradiated substrate under heat, forming the surface free radicals. So the reaction temperature is a sig-

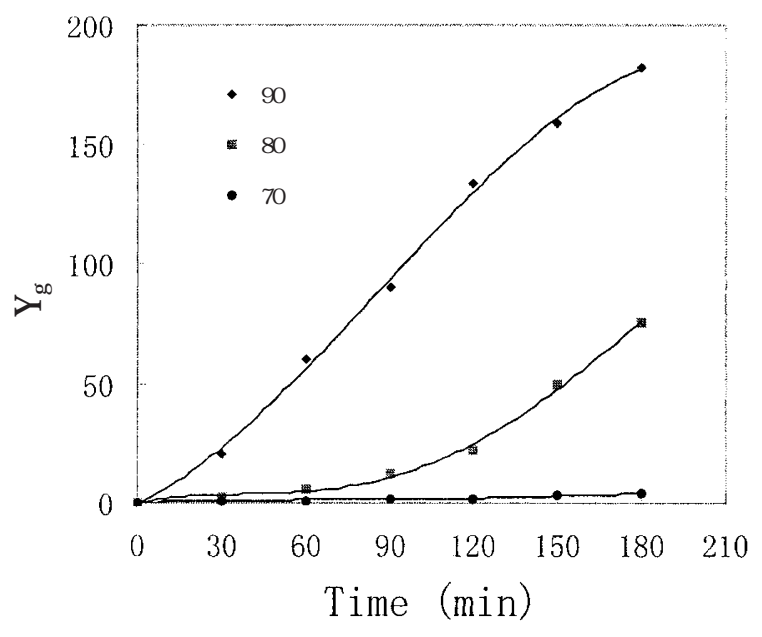

Figure 4. Effect of the reaction temperature on the grafting

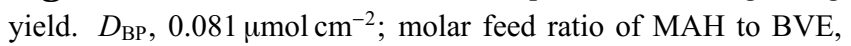
$1: 1$, the total concentration is $4 \mathrm{~mol} \mathrm{~L}^{-1}$; solvent, acetic anhydride.

nificant factor affecting the graft polymerization. The grafting performances under various temperatures are shown in Figure 4.

It can be seen from Figure 4 that the grafting yield at $70^{\circ} \mathrm{C}$ was very low, which indicated that the graft polymerization hardly took place at such reaction temperature. When the reaction temperature increased, the evolution of the graft polymerization became more and more smooth. At $80^{\circ} \mathrm{C}$, the graft polymerization could readily perform; at $90^{\circ} \mathrm{C}$, the grafting yield almost linearly increased along with the reaction time. From these facts it can be deduced that the desirable temperature to activate the "dormant" SBP groups to initiate graft polymerization is not less than $80^{\circ} \mathrm{C}$.

Because the surface free radicals are rooted on the substrate and the graft chain radicals are tied to the substrate, their mobility and vibration are largely inhibited. When the temperature increases, they will be largely activated. ${ }^{27}$ On the other hand, the diffusion of monomer towards the substrate is a crucial process for heterogeneous graft polymerization, and this process will speed up when the temperature increases. These are another two factors contributing to the favorable effect of higher temperature on graft polymerization.

\section{Effect of the Co-solvent Medium}

As illustrated above (Figures 3, 4, and Table II), the graft polymerization could readily undergo in the medium of acetic anhydride. However, if some acetone was added to form the co-solvent medium, the graft polymerization would remarkably speed up and high grafting yield could be obtained within short reaction time, as shown in Figure 5.

In the medium of $\mathrm{AAH} / 20 \mathrm{vol} \% \mathrm{AC}$, for example, the grafting yield reached as high as $180 \%$ within $1 \mathrm{~h}$, and 


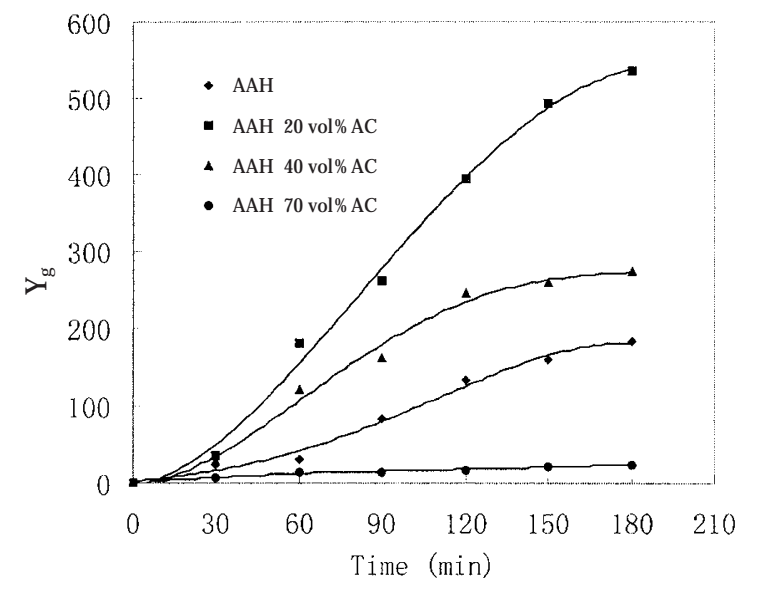

Figure 5. Performances of the graft polymerization in the medium of pure acetic anhydride and in the combination of acetic anhydride/acetone. $D_{\mathrm{BP}}, 0.081 \mu \mathrm{mol} \mathrm{cm}{ }^{-2}$; molar feed ratio of MAH to BVE, $1: 1$ the total concentration is $4 \mathrm{~mol} \mathrm{~L}^{-1}$; reaction temperature, $90^{\circ} \mathrm{C}$.

the corresponding density of the polar groups on the substrate was $49.5 \mu \mathrm{mol} \mathrm{cm}{ }^{-2}$; within $3 \mathrm{~h}$, the grafting yield could reach as high as $580 \%$. We observed that the grafted film with such high grafting yield became much larger (about 4-fold greater) and thicker than the original film, which indicated that the graft chains were situated not only on the surface but also on the subsurface or deep inside the substrate. The formation of the bulk graft chains was resulted from the existence of the inside SBP "dormant" groups (as discussed above) and the diffusion of monomer into the bulk of the substrate. On the other hand, the curves in Figure 5 also show that the $Y_{\mathrm{g}}$ values would notably decrease when the acetone content was too much. This is because of the severe vaporization of acetone (bp. $56^{\circ} \mathrm{C}$ ) at such reaction temperature, resulting in the great loss of the comonomer BVE (bp. $83^{\circ} \mathrm{C}$ ).

The favorable effect of acetone in the co-solvent medium can be interpreted from two aspects. 1) Acetone is a desirable solvent well affinitive towards PP substrate, which leads to the facile diffusion of monomer to the substrate. This result is just similar to that in the one-step photografting polymerization. ${ }^{19}$ 2) Acetone has better solubility for the component of the graft chains (MAH-alt-BVE) than acetic anhydride, so the graft chains can be thoroughly swelled in the presence of acetone (or other similar solvent, e.g., THF), resulting in the increasing mobility of the graft chain radicals. As a result of these two factors, the graft polymerization could be greatly promoted in the co-solvent medium, and consequently high grafting yield was obtained within short reaction time. The same solvent effect was once reported in J. Pavunec's literature too, ${ }^{28}$ and this may be a common phenomenon for heterogeneous graft polymerization.

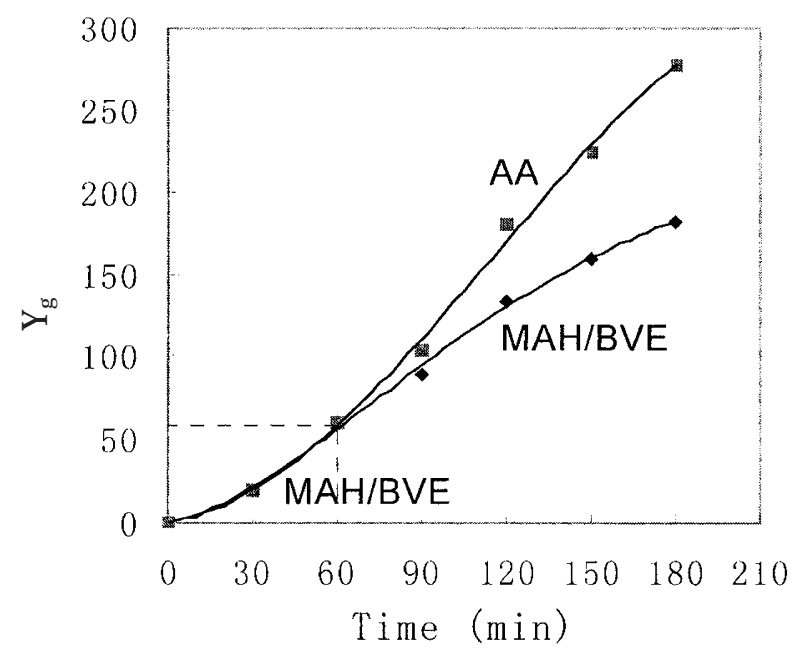

Figure 6. Successive graft polymerization of MAH/BVE and AA on the grafted PP films. The period of 0-60 min means the preparation of the first grafted PP film; feed ratio of MAH to BVE, $1: 1$, the total concentration is $4 \mathrm{~mol} \mathrm{~L}^{-1}$; AA concentration is $10 \mathrm{wt} \%$; solvent, acetic anhydride in the MAH/BVE system and water in the AA system; reaction temperature, $90^{\circ} \mathrm{C}$.

\section{Some Aspects of "Living" Graft Polymerization}

Originally, A.Btedzki and D.Braun reported that the synthesized telechelic oligomers terminated with phenoxydiphenylmethyl groups were effective initiators of further free radical polymerization of vinyl monomers. ${ }^{29}$ Enlightened by this precursory result, we once implanted the SBP groups, which have similar structure to the ones above, onto the film surface via photochemical process and realized the re-initiation of the graft chains. ${ }^{20}$ Later, H. M. Ma, C. N. Bowman, et al. also confirmed the same phenomenon by dynamic study. ${ }^{21}$

As for this particular grafting system, we put some previously grafted films into the MAH/BVE solution again and others were put into acrylic acid (AA) solution, to perform successive graft polymerization. The performances of the successive grafting are shown in Figure 6.

It can be seen from Figure 6 that the grafting yield of the grafted films increased continuously, which indicated that both AA and MAH/BVE readily underwent sequential graft polymerization on the grafted films. Since there was no additional initiator added into the grafting system, it is reasonable to deduce that the graft chains do have reinitiating ability. We observed that the grafting yield could keep increasing for more than $30 \mathrm{~h}$ at lower monomer concentration. The FT-IR spectra of these grafted films are shown in Figure 7.

In Figure 7, a distinct absorption peak appeared in the spectrum of the successively grafted PP film (d) compared with that of the first grafted PP film (b). Be- 


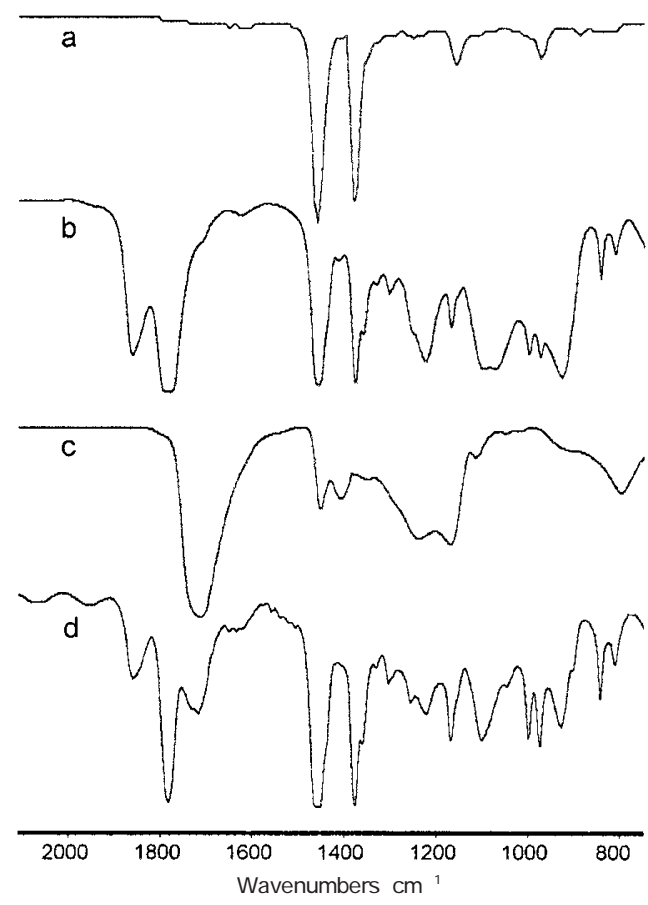

Figure 7. FT-IR spectra of a, blank PP film; $b$, first grafted PP film, c, Poly(acrylic acid), and d, successively grafted PP film (with AA monomer).

sides the anhydride group absorptions $\left(1785 \mathrm{~cm}^{-1}\right.$ and $\left.1860 \mathrm{~cm}^{-1}\right)$ and ether bond absorption $\left(1102 \mathrm{~cm}^{-1}\right)$, there still existed a carboxyl group at $1716 \mathrm{~cm}^{-1}$, indicating the existence of AA units. This means that AA was successfully grafted onto the existing graft chains, thus the block-structure graft chains were formed.

The reinitiating ability of the graft chains may be interpreted from two aspects. 1) As demonstrated above, the SBP radicals are not only difficult to couple each other but also hard to initiate homopolymerization, and their preferential tendency is to combine with surface free radicals in the absence of monomer or terminate with the growing chain radicals in the grafting process. ${ }^{20,21,30}$ On the other hand, the SBP groups endcapped on the graft chains can be easily activated and split off the substrate by heat; thus the graft chain radicals are reversibly formed and initiate sequential graft polymerization. Such process would perform again and again in the course of the reaction (as illustrated in Scheme 3), which may, to some extent, characterize the "living" radical polymerization.

Since the graft chains are covalently bonded onto the substrate, it is usually impossible to get pure graft chains from the grafted substrate. So a model initiator with SBP end groups was synthesized by photochemical reaction between BP and triethylamine (TEA), then a bulk polymerization of methyl acrylate (MA) was initiated by this model initiator with the concentration $0.3 \mathrm{~mol} \mathrm{~L}^{-1}$ at $90^{\circ} \mathrm{C}$. We observed that the molecular

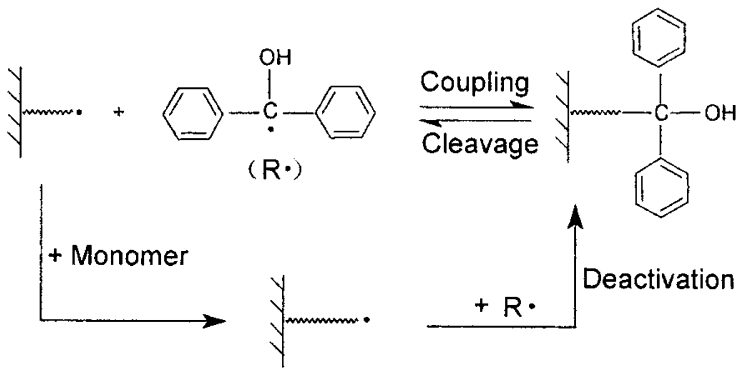

Scheme 3. Scheme of the reversible deactivation of SBP radicals to the propagating graft chains.

weight of PMA linearly increased at lower monomer conversion (e.g., $6.3 \times 10^{5}$ at $12.0 \%$ conversion and $8.8 \times 10^{5}$ at $32.2 \%$ conversion), and that the polydispersity index is relatively low $(1.36-1.59)$. This means that the polymerization initiated by the model initiator exhibited "living" characteristics to some extent. In other words, the reversible deactivation of SBP radicals to the propagating chains is relatively effective.

As for this surface grafting, the SBP groups can still be detected on the grafted films, which may also indicate that the termination pattern in the grafting process is mainly between the SBP radicals and the growing chain radicals. However, it should be noted that the graft polymerization based on SBP deactivation does not proceed in a strictly "living" pattern due to the heterogeneous reaction system.

2) In contrast to solution and bulk polymerization systems, the graft chain radicals are immobilized on the substrate, resulting in the steric limitation of a solid surface to which the chains are bonded, as well as the low mobility. So the graft chain radicals are not easy to be terminated by bimolecular reactions. This may be a physical factor contributing to the "living" graft polymerization.

With regard to the "living" graft polymerization, the weight increase of the grafted film can reflect the propagation of the graft chains to much extent, and the convenient means to trail the graft reaction is to measure the weight increase of the grafted film at regular intervals. The evolutions of the grafting yield and the average graft chain length were examined intensively, as illustrated in Figures $8 \mathrm{a}$ and $8 \mathrm{~b}$.

From Figure $8 \mathrm{a}$, it can be seen that at $90^{\circ} \mathrm{C}$, the grafting yield linearly increased on each pre-irradiated films along with the reaction time. And accordingly, the average graft chain length also linearly increased (Figure 8b). These results visibly revealed the "living" characteristic in the grafting process. Considering the approximate equality of $L_{\mathrm{c}}$ on the different preirradiated films at a given reaction time, this may suggest that the propagation rates of the graft chains were similar under the same grafting conditions, and that the 


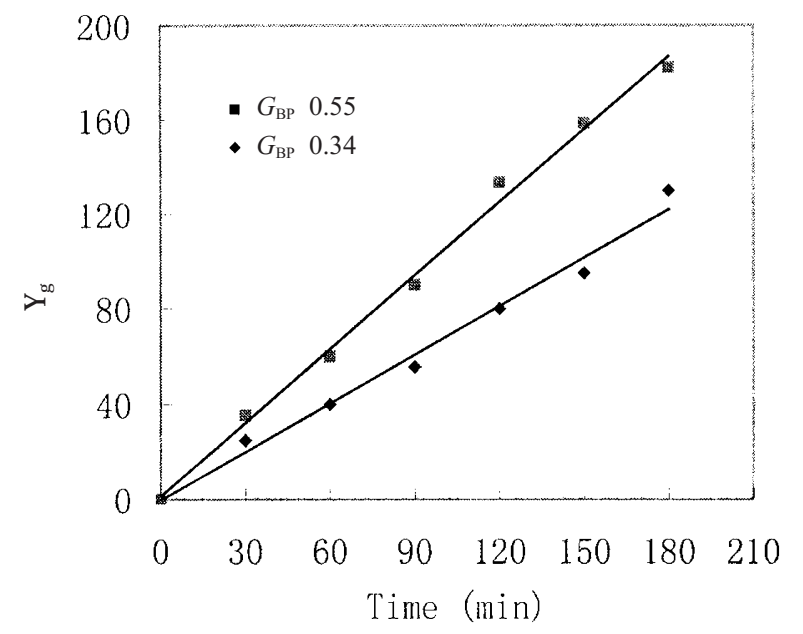

(a)

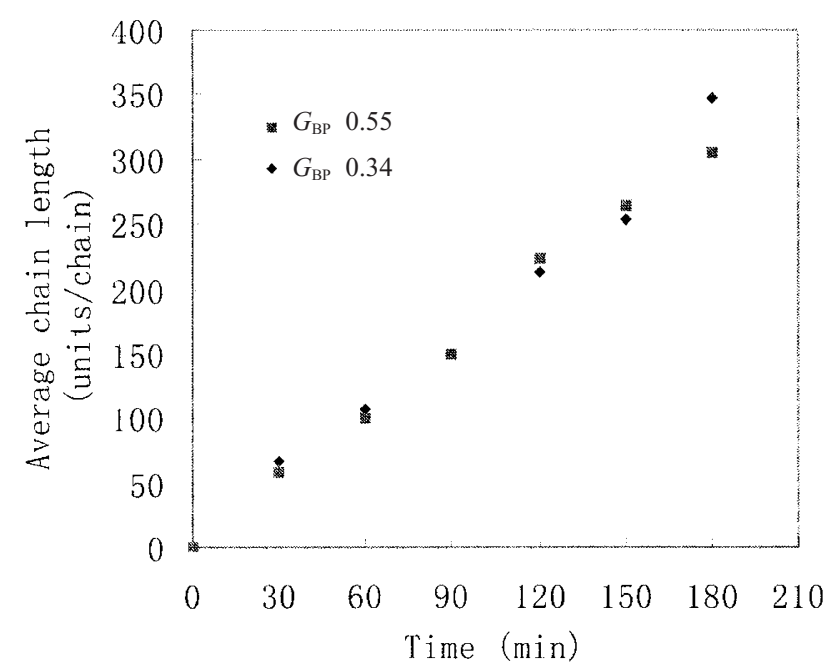

(b)

Figure 8. Evolutions of the grafting yield (a) and average grafting chain length (b) on the various pre-irradiated films. Molar feed ratio of MAH to BVE, 1:1, the total concentration is $4 \mathrm{~mol} \mathrm{~L}^{-1}$; solvent, acetic anhydride; reaction temperature, $90^{\circ} \mathrm{C}$.

difference of grafting yield on the various pre-irradiated films can be mainly attributed to the different density of the grafting points on the film surface.

In conclusion, the "living" characteristic in this grafting process may bring the facility to control the length and chemical structure of the graft chains. The grafting yield will further increase if the same monomer is successively added, or the block graft chains can be obtained if a different monomer is added.

\section{CONCLUSIONS}

(1) By means of introducing the electron donor monomer $n$-BVE, MAH can be facilely grafted onto the film surface.

(2) The grafting yield of two-step method can be much higher than that of one-step method.

(3) The grafting yield reaches the maximum value at
1:1 molar ratio of MAH to BVE, which is resulted from the formation of CTC between the binary monomers.

(4) The temperature required for the thermoactivating polymerization is around $80^{\circ} \mathrm{C}$; the solvents with good affinity towards the substrate and good solubility for the graft chains are fairly favorable to heterogeneous graft polymerization.

(5) Due to some chemical and physical factors, the grafting process exhibits some "living" characteristics to some extent, which may favor the control of the length and chemical structure of the graft chains.

Acknowledgment. This research has been supported by Special Funds for Major State Basic Research Projects (G1999064800) and the Chinese Outstanding Youth Foundation (20025415), which are gratefully acknowledged.

\section{REFERENCES}

1. C. Vasile and R. B. Seymour, Eds., "Handbook of Polyolefins", Marcel Dekker, New York, N.Y., 1993.

2. G. VerStrate, Encyclop. Polym. Sci. Engng., 6, 522 (1986).

3. N. G. Gaylord and M. Mehta, J. Appl. Polym. Sci., 33, 2549 (1987).

4. R. Singh, Prog. Polym. Sci., 17, 251 (1992).

5. J. Jagur-Grodzinski, Prog. Polym. Sci., 17, 361 (1992).

6. G. Natta and F. Severini, Makromol. Chem., 201, 119 (1968).

7. S. L. Lim and A. G. Fane, J. Appl. Polym. Sci., 41, 1609 (1990).

8. G. Marletta and S. Pignatoaro, Macromolecules, 24, 99 (1991).

9. T. Masuda and M. Kotoura, J. Appl. Polym. Sci., 43, 423 (1991).

10. C. L. Liu and Q. Wang, Polym. Mater. Sci. Eng., 15(3), 85 (1999).

11. S. Tazuke and H. Kimura, Makromol. Chem., 179, 2613 (1978).

12. W. T. Yang and B. Rånby, Polym. Bull., 37, 89 (1996).

13. J. P. Deng, W. T. Yang, and B. Rånby, J. Appl. Polym. Sci., 77, $1513(2000)$.

14. P. Y. Zhang and B. Rånby, J. Appl. Polym. Sci., 40, 1647 (1990).

15. H. Kubota and Y. Ogiwara, J. Appl. Polym. Sci., 43, 1001 (1991).

16. P. E. Gloor and Y. Tang, Polymer, 35, 1012 (1995).

17. R. M. Ho and A. C. Su, Polymer, 34, 3264 (1993).

18. H. Kubota, Y. Nobuyuki, and Y. Ogiwara, J. Appl. Polym. Sci., 39, 1231 (1990).

19. C. M. Xing, J. P. Deng, and W. T. Yang, Polym. J., 34, 801 (2002).

20. W. T. Yang and B. Rånby, Macromolecules, 29, 3308 (1996).

21. H. Ma, R. H. Davis, and C. N. Bowman, Macromolecules, 33, 331 (2000). 


\section{Surface Grafting Two-Step MAH $n$-BVE}

22. M. Ulbricht, H. Matuschewski, A. Oechel, and H. G. Hicke, J. Membr. Sci., 115, 31 (1996).

23. W. T. Yang and B. Rånby, Eur. Polym. J., 35, 1557 (1999).

24. M. Rätzsch, Prog. Polym. Sci., 27, 1195 (2002).

25. Y. Shirota and H. Mikawa, J. Macromol. Sci., -Rev. Macromol. Chem., C16(2), 129 (1977-1978).

26. C. M. Xing, J. P. Deng, and W. T. Yang, Polym. J., 34, 809
(2002).

27. W. T. Yang and B. Rånby, J. Appl. Polym. Sci., 62, 545 (1996).

28. J. Pavunec, M. Lazor, and Z. Manasek, J. Polym. Sci., Part C: Polym. Chem. Ed., 2, 113 (1967).

29. A. Btedzki and D. Braun, Macromol. Chem., 182, 1047 (1981).

30. W. T. Yang and B. Rånby, J. Appl. Polym. Sci., 62, 533 (1996). 